\title{
Comparative optimism about healthy eating
}

\author{
Gudrun Sproesser a, ${ }^{\text {, }}$, Verena Klusmann a , Harald T. Schupp ${ }^{\mathrm{b}}$, Britta Renner ${ }^{\mathrm{a}}$ \\ a University of Konstanz, Psychological Assessment and Health Psychology, Box 47, D-78457 Konstanz, Germany \\ ${ }^{\mathrm{b}}$ University of Konstanz, General Psychology, Box 36, D-78457 Konstanz, Germany
}

\section{A R T I C L E I N F O}

Keywords:

Healthy eating

Perceived healthiness

BMI

Intention

Unrealistic optimism

Self-perception

\begin{abstract}
A B S T R A C T
The present study investigated people's perception of their own as compared to their peers' healthy eating and related these perceptions to actual healthy eating, BMI, and subsequent healthy eating behavior. Data were collected within the framework of the longitudinal cohort study Konstanz Life Study (T1: N=770; T2: $N=510$ ). Our results demonstrated an optimistic bias on the group level. Specifically, people rated their own eating behavior as healthier on average than that of their average peers. This comparative optimism occurred even when actual healthy eating was unfavorable and BMI was high. However, it increased with actual healthy eating behavior. Importantly, optimistic perceptions were positively related to the intention to eat healthily and healthy eating six months later. Hence, the results suggest that an optimistic comparative view of one's own healthy eating is grounded in reality and boosts rather than deters subsequent health behavior. This implies that there might not be a need to reduce optimistic perceptions of healthy eating behavior.
\end{abstract}

\section{Introduction}

Most people tend to perceive themselves more favorably than others (e.g., Miles \& Scaife, 2003; Shepperd, Klein, Waters, \& Weinstein, 2013; Taylor \& Brown, 1988).

In a similar vein, Weinstein (1980) demonstrated in his agenda-setting study that, on average, people rated their own chances of experiencing positive events as above average and those of negative events as below average. On the individual level such an optimistic view might be accurate. On the group level, however,

it Acknowledgements: We thank Alexander Bürkle, María Moreno-Villanueva, Bettina Ott, Wolfgang Balig, Dennis Eichenbrenner and the Red Cross team, Simone BrunnerZilllikens and the Laboratory Brunner team, Ulrich Rüdiger, Julia Wandt and the press office of the University of Konstanz, Horst Frank, Ulrich Burchardt, Ralf Kleiner, Sabrina Schlaich, and Brigitte Kemmer-Przibylla from the City of Konstanz, Kirsten Schlüter for media cooperation with the Südkurier, Freda-Marie Hartung, Martina Gamp, Helge Giese, Filipa Gonçalves, Rita Juhasz, Fee Benz, Lukas Bernhart, Johanna Böttinger, Maike Bühler, Florian Ermark, Verena Friebe, Florian Furtwängler, Esther Gerdes, Kerstin Ha bgebauer, Jahan Heidari, Susanne Heinzelmann, Leonie Hepp, Verena Hoehne, Ursula Kirmse, Laura König, Hanna Kretschmann, Nicola Liguori, Cathrine Löber, Maximilian Mertel, Lisa Musculus, Friederike Papsdorf, Lena Schüler, Nino Sebastiani, Hendrik Sumpf, Katja Thömmes, Simon Vogt, Jens Winkler, and Angela Whale for their valuable support. Funding: This research was part of the EATMOTIVE project which was funded by the Federal Ministry of Education and Research (Grant 0315671, granted to Britta Renner and Harald Schupp). The funding sources had no involvement in study design; in the collection, analysis and interpretation of data; in the writing of the report; and in the decision to submit the article for publication. Conflict of interest: The authors declared that they had no actual or potential conflict of interest with respect to their authorship or the publication of this article including any financial, personal, or other relationships with other people or organizations.

* Corresponding author.

E-mail address: gudrun.sproesser@uni-konstanz.de (G. Sproesser). it represents an unrealistic optimistic bias: "If all people claim their changes of experiencing a negative event are less than average, they are clearly making a systematic error, thus demonstrating unrealistic optimism" (Weinstein, 1980, p. 806). Interestingly, such an unrealistic optimistic bias has also been found when children or adults evaluated the healthiness of their own eating behavior (Giese, Juhasz, Schupp, \& Renner, 2013; Paisley \& Sparks, 1998; Sparks, Shepherd, Wieringa, \& Zimmermanns, 1995). One important question concerning this phenomenon is whether people perceive their eating as healthier than their peers' eating regardless of their actual healthy or unhealthy eating habits. A second and maybe even more important question is which consequences arise from having an optimistic view of one's own eating regarding motivation for subsequent behavior.

\section{Perceptions of and actual healthy eating}

It is important to note that the optimistic bias consists of two components: The perception of oneself as compared to average others (e.g., Helweg-Larsen \& Shepperd, 2001; Perloff \& Fetzer, 1986; Renner, Gamp, Schmälzle, \& Schupp, 2015; Weinstein, 1980). Consequently, when investigating whether the extent of comparative optimism changes as a function of actual healthy eating, this change might be driven by people's self-perception or the perception of their average peers.

Regarding people's self-perception, studies suggest that people are relatively accurate in perceiving objective indicators (Chok, 2011; Renner, Gutierrez-Dona, Kwon, \& Schwarzer, 2009; Renner, Knoll, \& Schwarzer, 2000). For instance, people with lower body mass index (BMI) perceived their lifestyle as healthier (Chok, 2011) and their risk for cardiovascular diseases lower (Renner et al., 2000) as compared 
to people with higher BMI. BMI is an objective indicator which has been associated with healthy eating (e.g., Gao et al., 2008; Haslam \& James, 2005; but see Beydoun \& Wang, 2010; Kleiser, Mensink, Scheidt-Nave, \& Kurth, 2009). Moreover, the frequency of intake of healthy and unhealthy foods can be regarded as a more objective measure of healthy eating compared to people's general perception (see also Glanz, Brug, \& van Assema, 1997) because the first captures healthy eating behavior as defined by official nutrition guidelines.

When it comes to the relationship between objective indicators and people's peer-perception, past research reported mixed findings. In conditions of increasingly favorable objective indicators, people a) did not change their peer-perception (Chok, 2011), b) raised their peer-perception to the same degree as their self-perception (Renner et al., 2000), and c) raised their peer-perception even to a higher degree than their self-perception (Renner et al., 2009).

These patterns suggest three possible relationships between actual healthy eating and comparative optimism. First, with increasing actual healthy eating, people might increasingly perceive their eating as healthier than their peers' eating. In this case, people who actually eat healthily would have a greater optimistic view, grounded in reality (de Ridder, Fournier, \& Bensing, 2004; Gramling et al., 2008; Renner \& Schupp, 2011). Second, people might hold a constant optimistic view of their own compared to their peers' healthy eating regardless of actual healthy eating. Here, people might project different levels of selfperception onto their peers (Giese et al., 2013; Van Boven \& Loewenstein, 2003). Last, people's comparative optimistic perception of their healthy eating might be especially high if their actual eating habits are rather unhealthy. This pattern would speak in favor of a compensatory social downward comparison (Renner \& Schupp, 2011; Wilcox \& King, 2000).

\section{Consequences of an optimistic view of eating behavior}

Concerning the optimistic bias in risk perception, research suggests negative consequences for behavior change (e.g., Davidson \& Prkachin, 1997; Dillard, Midboe, \& Klein, 2009; Leikas, Lindeman, Roininen, \& Lähteenmäki, 2009; Shepherd, 2002; Weinstein, 1982). For instance, people who perceived their own risk as below average were less motivated to take precautions (Weinstein, 1982), showed less behavior change (Davidson \& Prkachin, 1997), and experienced more negative future events (Dillard et al., 2009). In a similar vein, Sparks et al. (1995) considered that an overestimation of one's own as compared to one's peers' healthy eating might prevent diet modification (see also Oenema \& Brug, 2003). Improving eating behavior, however, is a major societal task regarding the high and increasing prevalence of obesity (WHO, 2008). Hence, it is crucial to clarify the impact of an optimistic view of one's own as compared to one's peers' healthy eating behavior on the intention to eat healthily and subsequent eating behavior.

\section{The present study}

The present study examines people's perception of their own as compared to their peers' healthy eating behavior. The aims were: (1) to investigate whether people perceive their own eating behavior as healthier on average than an average peer's eating behavior; (2) to examine how people's self- and peer-perceptions are related to actual food intake and BMI; (3) to clarify the relevance of these perceptions to the intention to eat healthily; and (4) to investigate the impact of these perceptions on interindividual differences in healthy eating behavior six months later.

\section{Methods}

\section{Design and procedure}

Data were collected as part of the Konstanz Life Study, a longitudinal cohort study which was launched in spring 2012 (see Renner,
Sproesser, Klusmann, \& Schupp, 2012). The Konstanz Life Study was part of the EATMOTIVE project which was funded by the Federal Ministry of Education and Research (BMBF Grant 0315671, granted to B.R. and H.S.). At Wave 1, 1321 participants were recruited via flyers, posters, and newspaper articles. Waves 2 and 3 took place in autumn 2012 and spring 2013. Participants were invited to reattend via mail and phone calls. The three measurement points included the collection of blood samples, questionnaires, as well as a standardized checkup including anthropometric measures and functional and cognitive fitness tests. This study presents data measuring perceived healthiness from waves 2 (T1) and 3 (T2).

\section{Sample}

In total, 799 participants took part at T1. Of these, 29 were excluded due to missing data (see Analytical Procedure for details). Of the remaining 770 participants, 445 (58\%) were female and 201 (26\%) were living alone. At T1, the sample had a mean age of 47.7 years $(S D=17.5$, ranging from 19 to 87 years). The mean BMI was $24.8 \mathrm{~kg} / \mathrm{m}^{2}\left(S D=3.9\right.$, ranging from 17.3 to $\left.45.8 \mathrm{~kg} / \mathrm{m}^{2}\right)$. Participants had completed 15.8 years $(S D=2.4$, range from 8 to 20 years) of education on average and had a mean household income after taxes of $2250 €(M=6.93$ on an 11-point rating scale, $S D=2.35)$. Compared with German population data (Statistisches Bundesamt, 2014a, 2014b), this sample was 4 years older, comprised 7\% more females, and had a slightly lower average BMI (the average BMI of the German population is $26 \mathrm{~kg} / \mathrm{m}^{2}$ according to Microcensus data from 2009).

At T2, 543 participants re-attended the study. Of these, 33 had to be excluded due to missing data. Thus, the longitudinal sample comprised 510 participants (66\% of the cross-sectional sample). The longitudinal sample $(N=510)$ did not differ from the drop-out sample $(N=260)$ in terms of sex (57\% vs. $60 \%$ women, $\chi 2(1)=0.49, p=.488)$, living conditions ( $26 \%$ vs. $27 \%$ living alone, $\chi 2(1)=0.846, p=.862$ ), BMI ( 25.0 vs. $25.0 \mathrm{~kg} / \mathrm{m}^{2}, t(768)=-0.19, p=.848$ ), education ( 15.9 vs. 15.6 years, $t(743)=1.13, p=.290$ ), or study variables (i.e., actual food intake, perception of own and peers' healthy eating, intention to eat healthily, $|t| \mathrm{s}$ $(768) \leq 0.52, p s \geq .600)$. However, the longitudinal sample was 6 years older than the drop-out sample ( 49.1 vs. 43.3 years, $t(768)=4.44, p<.001$ ) and had a higher income; $2,250 €$ vs. $1,750 €, t(467.56)=4.53, p<.001$.

In terms of the representativeness of the longitudinal sample $(N=510)$ compared to the initial sample at $\mathrm{TO}(N=1321)$, the longitudinal sample did not differ from the drop-out sample $(N=811)$ in gender (57\% vs. $61 \%$ women, $\chi 2(1)=0.17, p=.185$ ), living conditions (26\% vs. $27 \%$ living alone, $\chi 2(1)=0.64, p=.654)$, BMI (25.0 vs. $24.8 \mathrm{~kg} / \mathrm{m}^{2}, t(1305)=1.05$, $p=.293$ ), or study variables (i.e., actual food intake, perception of own and peers' healthy eating, intention to eat healthily, $|t| \mathrm{s} \leq 0.55$, $p s \geq .580$ ). The longitudinal sample was on average 9 years older than the drop-out sample ( 49.1 vs. 40.4 years, $t(1311)=8.94, p<.001$ ), slightly better educated ( 15.9 vs. 15.5 years, $t(1269)=2.86, p=.004)$, and had a higher income; $2250 €$ vs. $1750 €, t(1148.09)=7.98, p<.001$.

All participants gave written informed consent prior to the collection of data and the ethical board of the University of Konstanz approved the study protocol. The procedures were performed in compliance with relevant laws and institutional guidelines. We strictly followed the German Psychological Society's (Deutsche Gesellschaft für Psychologie) guidelines for conducting psychological studies (see http://www.dgps.de/index.php?id=96422; see paragraph C.III). These are similar to those of the American Psychological Association. The study conforms to the Declaration of Helsinki.

\section{Measures}

\section{Actual food intake}

Actual food intake was assessed with a validated food frequency questionnaire at T1 (Winkler \& Döring, 1995, 1998; see also Sproesser, Strohbach, Schupp, \& Renner, 2011). Participants were 
asked how often on average they eat food items from 15 different selected food categories (e.g., whole meal products, vegetables, fruits, chocolate, cake, meat, and salty snacks), ranging from 1 (nearly once a day) to 6 (never). These 15 food categories were accumulated into a food frequency index reflecting dietary quality with a possible range of $0-30$. According to the recommendation of the German Nutritional Society and norm values for German samples on the basis of the WHO MONICA Augsburg Dietary Survey (see Winkler \& Döring, 1995; Winkler, Döring, \& Keil, 1991), scores of 13 or below indicate an unfavorable dietary pattern, scores greater than 13 and lower than 16 a regular dietary pattern, and scores of 16 or higher an optimal dietary pattern. In the cross-sectional (longitudinal) sample, an unfavorable dietary pattern was measured in 221 (152) participants. Of these, 122 (82) had normal or underweight (BMI below $25 \mathrm{~kg} / \mathrm{m}^{2}$ ). A regular dietary pattern was measured in 174 (112) participants, of whom 111 (75) had a BMI below $25 \mathrm{~kg} / \mathrm{m}^{2}$. Last, 375 (246) participants showed an optimal dietary pattern, of whom 220 (138) had a BMI below $25 \mathrm{~kg} / \mathrm{m}^{2}$.

\section{BMI}

Height and weight were measured following standardized procedures by trained research staff at T1. Participants wore light indoor clothing and were asked to take off their shoes. Height was measured to the nearest $0.1 \mathrm{~cm}$ using a wall-mounted stadiometer. Weight was measured using a digital scale (Omron Body Composition Monitor, BF511) to the nearest $0.1 \mathrm{~kg}$. BMI was calculated as weight in kilograms divided by height in meters squared.

\section{Perception of eating behavior}

In accordance with the indirect method of assessing an optimistic bias (see for example Perloff \& Fetzer, 1986; Renner \& Schupp, 2011), participants were asked to rate both their own eating behavior and that of an average peer of the same age and gender at T1 ("How do you rate your eating behavior?"; "How do you rate the eating behavior of an average person of your age and gender?"). Response scales ranged from 1 (very healthy) to 6 (very unhealthy). The coding of these two items was reversed. Furthermore, a difference score was calculated with positive values indicating that one's own eating habits were perceived as healthier than peer's.

\section{Intention to eat healthily}

The intention to eat healthily was assessed with a single item at T1 ("In future, I would like to eat....). The response scale ranged from 1 (unhealthily) to 7 (healthily).

\section{Healthy eating behavior}

Healthy eating behavior was assessed with a single item at T2 ("At the moment I eat..."). The response scale ranged from 1 (unhealthily) to 7 (healthily).

\section{Analytical procedure}

Statistical analyses were conducted using IBM SPSS statistics software (Version 21.0 for Windows). Participants were excluded from data analyses (1) if their weight or height was not measured at T1; and (2) if they filled in less than $75 \%$ of the questionnaires at T1 and T2. Missing data in the questionnaires at T1 and T2 were imputed using the Expectation Maximization algorithm in SPSS (Gold \& Bentler, 2000). Missing data in demographics were not imputed. Missing values were below $2 \%$ for all imputed variables and max. $3 \%$ for demographics. To examine whether study variables were normally distributed, normal quantile-quantile plots for standardized residuals were inspected (Judd \& McClelland, 1989).

To test our research questions Pearson correlations, two-tailed $t$-tests, and mixed ANOVAs including simple main effects were computed. Actual Dietary Pattern (unfavorable vs. regular vs. optimal), and $B M I$ (below $25 \mathrm{~kg} / \mathrm{m}^{2}$ vs. $25 \mathrm{~kg} / \mathrm{m}^{2}$ or higher) served as betweensubjects factors. The first ANOVA targeted Perception of Eating (self vs. peer) as within-subject factor. In the second ANOVA, IntentionBehavior (Intention to eat healthily at T1 vs. Healthy eating behavior at T2) served as within-subject factor to uncover differential (interactive) effects on intention compared to behavior. In control analyses, these two ANOVAs were extended by the betweensubjects factors Gender (female vs. male), Age (younger than 48 years vs. 48 years or older; median split), Living Conditions (living alone: yes vs. no), or Income (monthly household income after taxes below vs. above $2500 €$; median split). All tests were based on a 0.05 significance level. Effects of $d=0.2$ or $r=.1$ were regarded as small, effects of $d=0.5$ or $r=.3$ were regarded as medium, and effects of $d=0.8$ or $r=.5$ were regarded as large (Cohen, 1988). Effect sizes reported with Cohen's $d$ and correlation coefficient $r$ can be compared by the formula $r=d /\left(d^{2}+4\right)^{0.5}$ (Rosenthal, Rosnow, \& Rubin, 2000).

To secure our results with nonparametric statistics, Spearman correlations and Generalized Estimating Equations assuming a Poisson distribution were calculated. These analyses revealed highly similar findings as observed for Pearson correlations and ANOVA analysis. For brevity, results for Pearson correlations and ANOVA analysis are reported.

\section{Results}

\section{Self- vs. peer-perception of eating behavior}

Means, medians, standard deviations, and correlations between study variables are displayed in Table 1.

On average, participants perceived their own eating to be rather healthy $(M=4.25, S D=0.75)$. In comparison, they perceived their peers' eating behavior to be neither healthy nor unhealthy $(M=3.62$, $S D=0.67$ ). This difference on the group level indicates an optimistic bias, $t(769)=18.54, p<.001, d=.89$, with a large effect size (Cohen,

Table 1

Means, medians, standard deviations, and correlations between study variables $(N=770)$.

\begin{tabular}{|c|c|c|c|c|c|c|c|c|c|}
\hline & $M$ & $M d$ & $S D$ & 1 & 2 & 3 & 4 & 5 & 6 \\
\hline 1 Actual dietary pattern (T1) & 15.31 & 15.00 & 3.38 & - & & & & & \\
\hline 2 BMI (T1) & 24.81 & 24.15 & 3.91 & -.06 & - & & & & \\
\hline 3 Self-perception (T1) & 4.25 & 4.00 & 0.75 & $.37^{* *}$ & $-.21^{* *}$ & - & & & \\
\hline 4 Peer-perception (T1) & 3.62 & 4.00 & 0.67 & $.10^{* *}$ & .07 & $.13^{* *}$ & - & & \\
\hline 5 Difference self-peer (T1) & 0.63 & 1.00 & 0.94 & $.23^{* *}$ & $-.22^{* *}$ & $.71^{* *}$ & $-.61^{* *}$ & - & \\
\hline 6 Intention (T1) & 5.86 & 6.00 & 0.84 & $.30^{* *}$ & $-.07^{*}$ & $.41^{* *}$ & .06 & $.29^{* *}$ & - \\
\hline 7 Healthy eating $(\mathrm{T} 2)^{\mathrm{b}}$ & 5.12 & 5.00 & 1.16 & $.34^{* *}$ & $-.17^{* *}$ & $.57^{* *}$ & .06 & $.41^{* *}$ & $.49^{* *}$ \\
\hline
\end{tabular}

a Please note that correlations including this variable are not independent of correlations including participants' self- (3) and peer-perception (4).

b Values are for the longitudinal sample $(N=510)$.

${ }^{*} p<.05,{ }^{* *} p<.01$. 
1988). In total, only 67 participants (9\%) rated their own eating as less healthy than their peers' eating, whereas 417 participants (54\%) rated their eating as healthier than their peers' eating.

\section{Relation between perceptions, actual healthy eating, and BMI}

Participants perceived their eating as healthier the more favorable their actual dietary pattern was $(r=.37, p<.001)$, indicating relative accuracy. Similarly, participants' self-perception related significantly to their BMI $(r=-.21, p<.001)$. In contrast, participants' peer-perception increased only slightly with a more favorable dietary pattern $(r=.10, p=.008)$. The former was not significantly related to participants' BMI $(r=.07, p=.057)$. These two patterns resulted in a significant medium-sized association between the difference score of participants' self- and peer-perceptions and their actual dietary pattern. Specifically, the healthier participants' actual dietary pattern was, the healthier they rated their eating behavior as compared to their peers $(r=.23, p<.001)$. This comparative perception of healthy eating was also related to participants' BMI with a medium effect size $(r=-.22, p<.001)$.

To illustrate the effects of participants' actual dietary pattern and BMI on their self- and peer-perception, a $2 \times 3 \times 2$ ANOVA was computed (see Fig. 1a). We found a significant main effect of Perception of Eating, $F(1,764)=252.12, p<.001, \eta_{p}^{2}=.25$, and of Actual Dietary Pattern, $F(2,764)=34.46, p<.001, \eta_{p}^{2}=.08$. Furthermore, significant interactions occurred between the factors Perception of Eating and Actual Dietary Pattern as well as between Perception of Eating and BMI, $F(2,764)=13.73, p<.001, \eta_{p}^{2}=.04$ and $F(1,764)=17.41$, $p<.001, \eta_{p}^{2}=.02$. There were no further significant effects, $F \mathrm{~s}(2$, $764) \leq 1.44, p s \geq .237$.

First, simple main effects replicated the presence of an optimistic bias on the group level. Regardless of dietary pattern or BMI, participants perceived their own eating behavior to be healthier than their peers' eating behavior, $F s(1,764) \geq 4.35, p s \leq .037, \eta^{2} s \geq .01$.

Second, participants' perception of their own eating behavior increased with increased healthiness in their dietary pattern. This increase was significant in both BMI groups, $F s(2,764) \geq 21.44$, $p s \leq .001, \eta^{2} s \geq .05$. Similarly, participants with a BMI below $25 \mathrm{~kg} /$ $\mathrm{m}^{2}$ perceived their eating behavior to be healthier than participants with a BMI of $25 \mathrm{~kg} / \mathrm{m}^{2}$ or higher. This was especially true for participants with an unfavorable dietary pattern, $F(1,764)=4.67, p=.031$, $\eta^{2}=.01$. The simple main effect of BMI did not reach significance in other dietary pattern groups, $F s(1,764) \leq 3.69, p s \geq .055$. These results confirm relative accuracy in participants' self-perception, in line with correlational results.

Third, participants' perception of their peers' eating behavior slightly increased in participants with lower BMI the more favorable their dietary pattern was, $F(2,764)=3.82, p=.022, \eta^{2}=.01$. In participants with higher BMI, the peer-perception was independent of dietary pattern, $F(2,764)=1.67, p=.189$. Likewise, BMI was not significantly related to participants' peer-perception across all three dietary patterns, $F \mathrm{~s}(1,764) \leq 3.76, p s \geq .053$. Hence, peerperception was rather unrelated to actual healthy eating and BMI.

Taken together, these results demonstrate that participants' optimistic view of their healthy eating increased with actual healthy eating and lower BMI. Specifically, the group demonstrating the largest optimism regarding their own as compared to their peers' eating was the one with the most favorable actual healthy eating and lower BMI (see Fig. 1a). In contrast, the group with the least favorable diet and a higher BMI, held the smallest optimistic view of their eating.

To investigate potential confounders, we first examined the relationship between actual dietary pattern, BMI, and demographics. Women had a healthier dietary pattern than men, $M=15.76, S E=0.16$

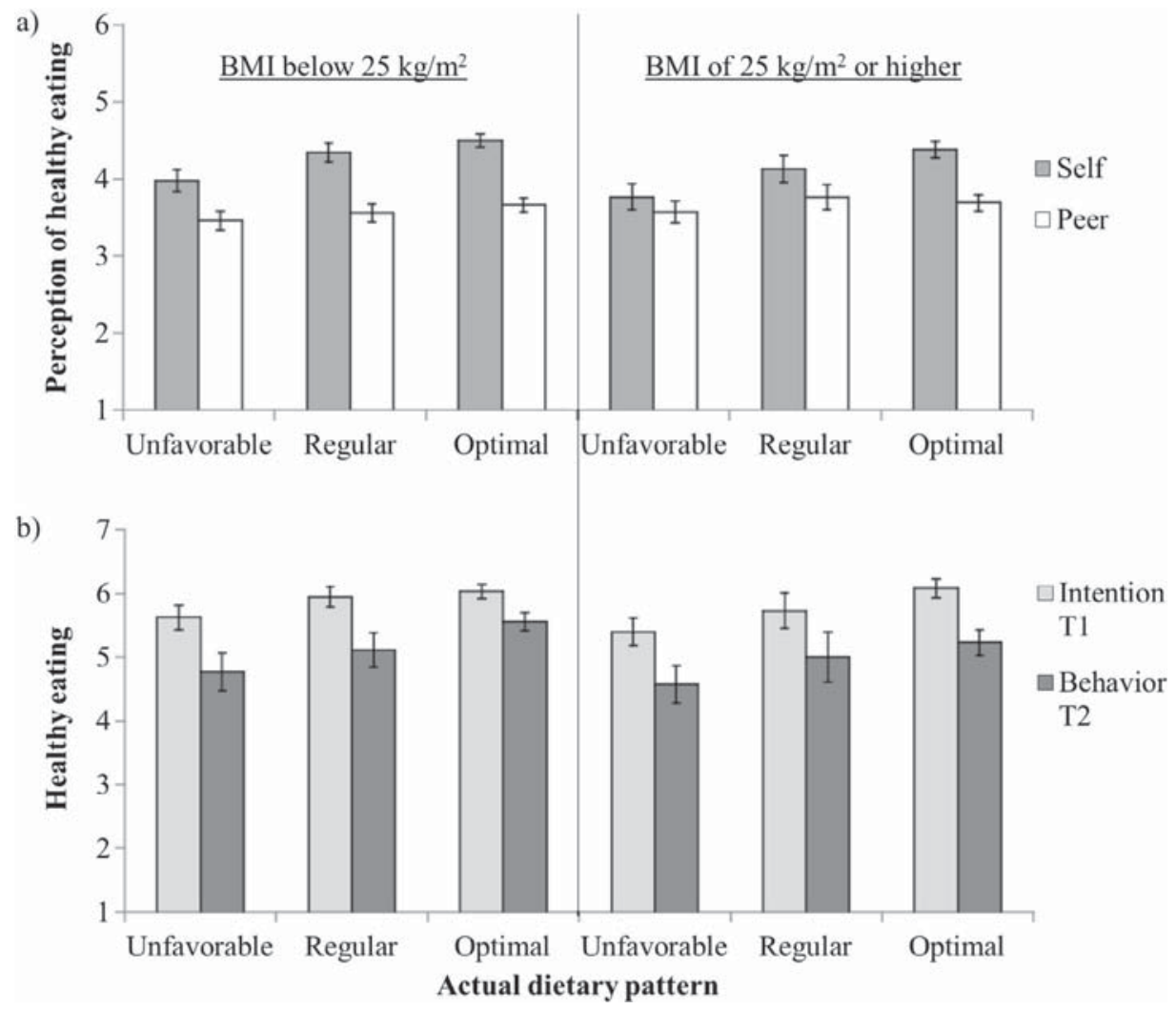

Fig. 1. (a) Perception of own and peers' eating as a function of actual dietary pattern and BMI at T1 $(N=770)$. (b) Intention to eat healthily at T1 and healthy eating at T2 as a function of actual dietary pattern and BMI at $\mathrm{T} 1(N=510)$. Error bars indicate $95 \%$ confidence intervals. 
vs. $M=14.70, S E=0.19, t(768)=4.37, p<.001, d=.32$, and a lower BMI, $M=24.24, S E=0.19$ vs. $M=25.60, S E=0.20, t(768)=-4.85$, $p<.001, d=-.35$. Furthermore, actual dietary pattern and BMI were significantly related to age $(r=.26$ and $r=.22$, $p s<.001)$. Older participants ate healthier than younger participants and had higher BMIs. In contrast, participants living together with other people did not differ from participants living alone in terms of dietary pattern, $M=15.25, S E=0.14$ vs. $M=15.44, S E=0.26, t(322.30)=-0.65, p=.515$, or BMI, $M=24.76, S E=0.16$ vs. $M=24.86, S E=0.29, t(759)=-0.31$, $p=.755$. Dietary pattern and BMI were also unrelated to participants' income $(r=.03, p=.412$ and $r=.05, p=.174)$.

Second, including Gender, Age, Living Conditions, or Income in the mixed ANOVA displayed in Fig. 1a revealed unchanged results. Living Conditions and Income had no significant effects, $F \mathrm{~s}<2.93$, $p s>.054$. However, an additional significant main effect of Gender, $F(1,758)=22.79, p<.001, \eta^{2}=.03$, and Age, $F(1,758)=20.46, p<.001$, $\eta^{2}=.03$, emerged as well as a significant interaction of Perception of Eating $\times$ Gender, $F(1,758)=16.37, p<.001, \eta^{2}=.02$, and of Perception of Eating $\times$ Actual Dietary Pattern $\times$ Age, $F(2,758)=5.19$, $p=.006, \eta^{2}=.01$.

Concerning the impact of gender, men rated their own eating behavior as healthy as women $\left(M_{\text {self }}=4.15, S E=0.04\right.$ vs. $M_{\text {self }}=4.20$, $S E=0.04 ; F(1,758)=0.67, p=.413)$. However, men showed a larger optimistic bias by rating their peers' eating behavior more unhealthy than women $\operatorname{did}\left(M_{\text {peer }}=3.44, S E=0.04\right.$ vs. $M_{\text {peer }}=3.78$, $\left.S E=0.04 ; F(1,758)=43.36, p<.001, \eta^{2}=.05\right)$.

Concerning the impact of age, younger participants rated their own and their peer's eating behavior less healthy than older participants (self: $M=4.11, S E=0.04$ vs. $M=4.38, S E=0.04$; peer: $M=3.52, S E=0.03$ vs. $M=3.71, S E=0.03)$. Inspecting the interaction of Perception of Eating $\times$ Actual Dietary Pattern $\times$ Age showed that the decrease in participants' self-perception with decreasingly favorable dietary pattern was more pronounced in younger participants.

Relation between an optimistic view, intention to eat healthily, and subsequent healthy eating behavior

The results show that perceiving one's own eating behavior healthier than that of an average peer was not detrimental for participants' intention to eat healthily but showed a medium-sized positive correlation $(r=.29, p<.001$; see Table 1$)$. Moreover, an optimistic view of one's own eating behavior was positively related to healthy eating six months later with a large effect size $(r=.41$, $p<.001)$.

To exemplify the absolute level of intention and healthy eating at six months in the groups whose comparative perception of healthy eating is displayed in Fig. 1 a, a mixed $2 \times 3 \times 2$ ANOVA was performed (see Fig. 1b). This analysis revealed a significant main effect of the within-subject factor Intention-Behavior as well as of the between-subjects factors Actual Dietary Pattern and BMI, $F(1$, $504)=234.55, p<.001, \eta^{2}=.32 ; F(2,504)=28.75, p<.001, \eta^{2}=.10$; $F(1,504)=4.72, p=.030, \eta^{2}=.01$. No interaction term was significant, Fs $\leq 2.83$, ps $\geq .060$.

Simple main effect analyses revealed, first, that the intention to eat healthily was higher than healthy eating behavior across all subgroups, $F s(1,504) \geq 18.40$, $p s \leq .001, \eta^{2} s \geq .04$. Second, the healthier participants' dietary pattern was, the higher was their intention to eat healthily and the healthier was their eating behavior six months later in both BMI groups, $F s(2,504) \geq 7.26, p s \leq .001, \eta^{2} s \geq .03$. Third, participants with higher BMI tended to have lower intentions to eat healthily at T1 and tended to show less healthy eating at T2. Inspecting the subgroups revealed that especially participants with higher BMI and an optimal dietary pattern reported less healthy eating at T2 than participants with lower BMI and an optimal dietary pattern, $F(1,504)=5.22, p=.023, \eta^{2}=.01$. The remaining simple main effects of BMI did not reach significance, $F s(1,504) \leq 3.07, p s \geq .080$.

Comparing Fig. 1a and b demonstrates that the group with the largest optimistic view (people with lower BMI and an optimal dietary pattern) was among those with the highest intention to eat healthily. Moreover, this group reported the healthiest eating behavior half a year later. In contrast, the group with the smallest self-peer discrepancy (people with higher BMI and an unfavorable dietary pattern) had the lowest intention to eat healthily and reported the least healthy eating at six months.

In a next step, potential confounders were investigated. Including Gender, Age, Living Conditions, or Income in the mixed ANOVA displayed in Fig. 1b yielded unchanged results. Only in the ANOVA including Gender, the main effect of BMI was only marginally significant, $F(1,498)=3.57, p=.060, \eta^{2}=.01$. Additionally, in the ANOVA including Age the interaction of Intention-Behavior $\times$ Actual Dietary Pattern $\times$ BMI became significant, $F(2,498)=3.10, p=.046, \eta^{2}=.01$. Inspecting Fig. 1b shows that participants with low BMI and optimal dietary pattern, who had the largest optimistic view of their eating, displayed the smallest gap between their intention and behavior at $\mathrm{T} 2$.

Age and living conditions had no significant effects, $F s<2.27$, ps $>.132$. However, there was a significant main effect of Gender, with men having a lower intention to eat healthily $(M=5.58, S E=0.06$ vs. $\left.M=5.89, S E=0.05 ; F(1,498)=7.19, p=.008, \eta^{2}=.01\right)$. Moreover, a significant main effect of Income occurred, $F(1,491)=3.98$, $p=.047, \eta^{2}=.01$, a significant Intention-Behavior $\times$ Income interaction, $F(1,491)=4.69, p=.031, \eta^{2}=.01$, and a significant IntentionBehavior $\times \mathrm{BMI} \times$ Income interaction, $F(1,491)=9.14, p=.003, \eta^{2}=.02$. Inspecting this interaction showed that especially people with higher income ate healthier at T2 when their BMI was below $25 \mathrm{~kg} / \mathrm{m}^{2}$ as compared to people with lower income and low BMI $(M=5.40$, $S E=0.07$ vs. $M=5.00, S E=0.12$ ).

\section{Discussion}

The present study comprises the first systematic investigation of people's perception of their own as compared to their peers' healthy eating, relating these perceptions to actual healthy eating, BMI, and subsequent behavior. Our results demonstrated an optimistic bias on the group level. Specifically, people rated their own eating behavior as healthier on average than that of their average peers. This comparative optimism was larger the more favorable actual healthy eating and the lower BMI was. Moreover, it was also positively related to the intention to eat healthily and healthy eating six months later. Hence, these results show that an optimistic comparative view of one's own healthy eating is grounded in reality and associated with an enhanced motivation for subsequent healthy eating.

\section{Optimistic perceptions: grounded in reality}

Our finding of an optimistic bias in eating behavior on the group level is in accordance with previous research (Giese et al., 2013; Paisley \& Sparks, 1998; Sparks et al., 1995). Moreover, in line with findings showing that optimistic beliefs are grounded in reality (Gramling et al., 2008), we found that comparative optimism increased relative to healthier actual food intake and to lower BMI. However, people still rated their own eating behavior to be healthier than their peers' eating when actual healthy eating was unfavorable and BMI was high. Disentangling people's self- and peer-perception demonstrated that people were relatively accurate in perceiving actual healthy eating (see also Chok, 2011; Renner et al., 2000, 2009). Interestingly, actual healthy eating and BMI did not affect people's peer-perception (see also Chok, 2011). 
Within an alternative assumption, we considered that although people view themselves as superior, they might project different levels of their self-perception onto their peers (Giese et al., 2013; Renner et al., 2000; Van Boven \& Loewenstein, 2003). However, we only found a slightly more positive perception of peers' eating with increasing actual healthy eating in people with lower BMI and the effect was small. Furthermore, people with higher BMI showed rather the opposite pattern to this alternative assumption: Whereas people with higher body weight rated their own eating as less healthy than people with lower body weight, the former tended to rate their peers' eating as healthier than the latter. Given that previous research has demonstrated that people underestimate their peers' healthy eating and overestimate their unhealthy behaviors (Lally, Bartle, \& Wardle, 2011; Perkins, 2007), people with higher BMI tended to be more accurate in their peer-perception. This might result from enhanced attention to others' eating behavior in order to ascertain an eating pattern that would help them normalize their weight. To sum up, our results argue for a largely independent perception of one's own eating behavior and that of one's peers.

Our results do not provide support for a compensatory social downward comparison in the domain of healthy eating behavior. Although such a downward comparison has been suggested as operating in older adults to maintain well-being despite age-related health decline (Renner et al., 2009; Wilcox \& King, 2000), people with a poor diet or higher BMI did not compensate for their less favorable self-perception by downgrading their peers. This supports the notion that people perceive information about their eating behavior and body weight in a non-defensive way.

Ruling out the explanation that an optimistic view of eating behavior results from a defense when actual behavior is unfavorable poses the question of which determinants might underlie the phenomenon. Motivational (e.g., self-enhancement) and cognitive processes (e.g., egocentric bias) have been discussed (see Renner \& Schupp, 2011 for an overview). Pronin (2008) argued that people judge themselves rather on their good intentions whereas they judge others by their less-good behavior.

\section{An optimistic view as path to subsequent healthy eating behavior}

Classical findings showed that unrealistic optimism in risk perception is detrimental to motivation and subsequent health behavior (e.g., Davidson \& Prkachin, 1997; Weinstein, 1982). In contrast, our study demonstrated that an optimistic perception of eating behavior is positively related to the intention and subsequent healthy eating behavior.

How could this health-promoting relation between optimistic perceptions and subsequent behavior be explained? On the one hand, the extent of people's optimistic view was mostly driven by their self-perception with a rather constant peer-perception. On the other hand, actual healthy eating was translated into these self-perceptions. As previous studies have shown that past behavior is related to intention and subsequent behavior (e.g., Conner, Norman, \& Bell, 2002; Gutierrez-Dona, Lippke, Renner, Kwon, \& Schwarzer, 2009; Houben, Nederkoorn, \& Jansen, 2012), it might have been actual eating behavior, mirrored in people's self-perception, which led to higher intention and subsequent healthy eating behavior.

The found differences in healthy eating are likely to be physiologically relevant for people's health given the significant impact of food habits on health (e.g., Ford et al., 2009). Romaguera et al. (2011) showed that a two-point increment on a 19 point dietary healthiness scale was associated with a $4 \%$ reduction in the risk of type 2 diabetes. Assuming that the assessment of healthy eating of Romaguera et al. (2011) is comparable to the assessment of healthy eating in our study, one can transfer the effect of healthy eating on diabetes to our study. Specifically, the mean 1 point higher healthy eating of the group with the largest optimism (optimal dietary pattern and low BMI) as compared to the group with the lowest optimism (unfavorable dietary pattern and high BMI; see Fig. 1b), would correspond to a $6 \%$ lower risk of diabetes of the first group.

In the present study, we related people's self- and peer-perception of healthy eating to the objective indicator BMI and the frequency of eating healthy or unhealthy foods. However, evidence about the relationship between healthy eating and BMI is mixed, with some papers pointing to a negative relationship (e.g., Gao et al., 2008; Haslam \& James, 2005) whereas others do not find an association (e.g., Beydoun \& Wang, 2010; Kleiser et al., 2009). In our study, people with higher BMI might have eaten as healthily as people with lower BMI, with their higher body weight resulting, for example, from a lower level of energy expenditure or unhealthy eating in the past. In this case, overweight people would have wrongly rated their eating behavior less healthily. This would be of concern regarding our finding that a lower self-perception was associated with a lower intention and less healthy eating at six months.

\section{Limitations}

Some limitations of the present study need to be taken into account. First, we cannot definitely determine whether what we called optimistic bias is actually a bias since it is unknown whether our sample was representative in terms of dietary healthiness. Second, while our cross-sectional and longitudinal sample was representative of the German population in terms of gender, BMI, and age (Statistische Bundesamt, 2014a, 2014b), findings are limited by drop-outs. This could have led to a selection bias leaving, for example, people with higher motivation in the longitudinal sample. The issue needs to be also considered in interpreting the medium to large effect sizes of the main findings reported in the study. Moreover, the longitudinal sample was older, better educated, and had a higher income than the dropout sample. As higher SES groups were demonstrated to have healthier dietary patterns and lower BMIs (e.g., Pechey et al., 2013; Roskam \& Kunst, 2008), future research needs to determine whether similar findings are obtained in lower SES groups.

Third, we cannot draw conclusions on causality because data have been cross-sectional or static (Renner, Hankonen, Ghisletta, \& Absetz, 2012). Fourth, healthy eating behavior at T2 was assessed with a single item. This parallel assessment of intention at $\mathrm{T} 1$ and healthy eating behavior at $\mathrm{T} 2$ was chosen to directly compare possible differential effects on intention and behavior. However, we cannot rule out the possibility that this measure might assess a combination of people's actual healthy eating and their perception of healthy eating. Hence, future research needs to address these points to secure the found results.

\section{Conclusion}

The present study demonstrated a pervasive optimistic view of one's own as compared to one's peers' healthy eating. Furthermore, this optimistic comparative perception of healthy eating was positively related to actual healthy eating, the intention to eat healthily and healthy eating six months later. Thus, our findings suggest that this comparative optimism is grounded in reality and boosts rather than deters subsequent healthy eating behavior. Future research allowing for causal inferences is needed before considering the promotion of comparative optimism as a means to enhance healthy eating behavior. In sum, based on the present findings, there seems to be no need to reduce optimistic perceptions of one's healthy eating behavior in intervention programs designed to reduce body weight or improve healthy eating. 


\section{References}

Beydoun, M. A., \& Wang, Y. (2010). Pathways linking socioeconomic status to obesity through depression and lifestyle factors among young US adults. Journal of Affective Disorders, 123, 52-63. doi:10.1016/j.jad.2009.09.021.

Chok, T. M. (2011). The influence of body mass index, sex, and race on college students' optimistic bias for lifestyle healthfulness. Journal of Nutrition Education and Behavior, 43, 331-338. doi:10.1016/j.jneb.2010.09.016.

Cohen, J. (1988). Statistical power analysis for the behavioral sciences. Hillsdale, NJ: Erlbaum.

Conner, M., Norman, P., \& Bell, R. (2002). The theory of planned behavior and healthy eating. Health Psychology, 21, 194-201. doi:10.1037//0278-6133.21.2.194.

de Ridder, D., Fournier, M., \& Bensing, J. (2004). Does optimism affect symptom report in chronic disease? What are its consequences for self-care behaviour and physical functioning? Journal of Psychosomatic Research, 56, 341-350. doi:10.1016/s00223999(03)00034-5.

Davidson, K., \& Prkachin, K. (1997). Optimism and unrealistic optimism have an interacting impact on health-promoting behavior and knowledge changes. Personality and Social Psychology Bulletin, 23, 617-625. doi:10.1177| 0146167297236005.

Dillard, A. J., Midboe, A. M., \& Klein, W. M. P. (2009). The dark side of optimism. Unrealistic optimism about problems with alcohol predicts subsequent negative event experiences. Personality and Social Psychology Bulletin, 35, 1540-1550. doi: $10.1177 / 0146167209343124$

Ford, E. S., Bergmann, M. M., Kroeger, J., Schienkiewitz, A., Weikert, C., \& Boeing, H. (2009). Healthy living is the best revenge findings from the European prospective investigation into cancer and nutrition-potsdam study. Archives of Internal Medicine, 169, 1355-1362.

Gao, S. K., Beresford, S. A. A., Frank, L. L., Schreiner, P. J., Burke, G. L., \& Fitzpatrick, A. L. (2008). Modifications to the Healthy Eating Index and its ability to predict obesity. The multi-ethnic study of atherosclerosis. American Journal of Clinical Nutrition, 88, 64-69.

Giese, H., Juhasz, R., Schupp, H., \& Renner, B. (2013). Kann man Popularität und Freundschaft essen? Der Zusammenhang zwischen wahrgenommener Ernährung populärer und sympathischer Kinder und dem eigenen Ernährungsverhalten von Kindern [Can you eat popularity and friendship? The relationship between the perceived eating behavior of popular and likeable children and the individual eating behavior of children]. Zeitschrift für Gesundheitspsychologie, 21, 71-81. doi:10.1026/0943-8149/a000092.

Glanz, K., Brug, J., \& van Assema, P. (1997). Are awareness of dietary fat intake and actual fat consumption associated? A Dutch-American comparison. European Journal of Clinical Nutrition, 51, 542-547. doi:10.1038/sj.ejcn.1600442.

Gold, M. S., \& Bentler, P. M. (2000). Treatments of missing data. A Monte Carlo comparison of RBHDI, iterative stochastic regression imputation, and expectation-maximization. Structural Equation Modeling, 7, 319-355. doi:10.1207| s15328007sem0703_1.

Gramling R. Klein, W., Roberts, M., Waring M. E. Gramling D. \& Eaton, C. B. (2008). Self-rated cardiovascular risk and 15-year cardiovascular mortality. Annals of Family Medicine, 6, 302-306. doi:10.1370/afm.859.

Gutierrez-Dona, B., Lippke, S., Renner, B., Kwon, S., \& Schwarzer, R. (2009). Self-efficacy and planning predict dietary behaviors in Costa Rican and South Korean women. Two moderated mediation analyses. Applied Psychology-Health and Well-Being, 1,91-104. doi:10.1111/j.1758-0854.2008.01001.x.

Haslam, D. W., \& James, W. P. T. (2005). Obesity. Lancet, 366, 1197-1209. doi:10.1016/ s0140-6736(05)67483-1.

Helweg-Larsen, M., \& Shepperd, J. A. (2001). Do moderators of the optimistic bias affect personal or target risk estimates? A review of the literature. Personality and Social Psychology Review, 5, 74-95. doi:10.1207/s15327957pspr0501_5.

Houben, K., Nederkoorn, C., \& Jansen, A. (2012). Too tempting to resist? Past success at weight control rather than dietary restraint determines exposure-induced disinhibited eating. Appetite, 59, 550-555. doi:10.1016/j.appet.2012.07.004.

Judd, C. M., \& McClelland, G. H. (1989). Data analysis. A model-comparison approach. San Diego, CA: Harcourt, Brace, Jovanovich.

Kleiser, C., Mensink, G. B. A., Scheidt-Nave, C., \& Kurth, B.-M. (2009). HuSKY. A healthy nutrition score based on food intake of children and adolescents in Germany. British Journal of Nutrition, 102, 610-618. doi:10.1017/s0007114509222689.

Lally, P., Bartle, N., \& Wardle, J. (2011). Social norms and diet in adolescents. Appetite, 57, 623-627. doi:10.1016/j.appet.2011.07.015.

Leikas, S., Lindeman, M., Roininen, K., \& Lähteenmäki, L. (2009). Who is responsible for food risks? The influence of risk type and risk characteristics. Appetite, 53, 123-126. doi:10.1016/j.appet.2009.05.003.

Miles, S., \& Scaife, V. (2003). Optimistic bias and food. Nutrition Research Reviews, 16, 3-19. doi:10.1079/nrr200249.

Oenema, A., \& Brug, J. (2003). Exploring the occurrence and nature of comparison of one's own perceived dietary fat intake to that of self-selected others. Appetite, 41, 259-264. doi:10.1016/s0195-6663(03)00103-x.

Paisley, C. M., \& Sparks, P. (1998). Expectations of reducing fat intake. The role of perceived need within the theory of planned behaviour. Psychology \& Health, 13, 341-353. doi:10.1080/08870449808406755.
Pechey, R., Jebb, S. A., Kelly, M. P., Almiron-Roig, E., Conde, S., Nakamura, R., et al. (2013). Socioeconomic differences in purchases of more vs. less healthy foods and beverages. Analysis of over 25,000 British households in 2010. Social Science E Medicine, 92, 22-26. doi:10.1016/j.socscimed.2013.05.012.

Perkins, H. W. (2007). Misperceptions of peer drinking norms in Canada. Another look at the "reign of error" and its consequences among college students. Addictive Behaviors, 32, 2645-2656. doi:10.1016/j.addbeh.2007.07.007.

Perloff, L. S., \& Fetzer, B. K. (1986). Self-other judgments and perceived vulnerability to victimization. Journal of Personality and Social Psychology, 50, 502-510. doi:10.1037//0022-3514.50.3.502.

Pronin, E. (2008). How we see ourselves and how we see others. Science, 320(5880), 1177-1180. doi:10.1126/science.1154199.

Renner, B., Gamp, M., Schmälzle, R., \& Schupp, H. T. (2015). Health risk perception, In J. D. Wright (Hrsg.) (Ed.), The international encyclopedia of social and behavioral sciences (2nd ed.). Oxford, England: Elsevier.

Renner, B., Gutierrez-Dona, B., Kwon, S., \& Schwarzer, R. (2009). Risk perception across age groups and countries. Becoming more vulnerable but being still invincible. Psychology \& Health, 24, 336.

Renner, B., Hankonen, N., Ghisletta, P., \& Absetz, P. (2012). Dynamic psychological and behavioral changes in the adoption and maintenance of exercise. Health Psychology, 31, 306-315. doi:10.1037/a0025302.

Renner, B., Knoll, N., \& Schwarzer, R. (2000). Age and body weight make a difference in optimistic health beliefs and nutrition behaviors. International Journal of Behavioral Medicine, 7, 143-159.

Renner, B., \& Schupp, H. (2011). The perception of health risks. In H. S. Friedman (Ed.), Oxford handbook of health psychology (pp. 637-665). New York: Oxford University Press.

Renner, B., Sproesser, G., Klusmann, V., \& Schupp, H. (2012). Die Konstanzer Life-Studie [The Konstanz Life Study]. Adipositas, 6, 123-124.

Romaguera, D., Guevara, M., Norat, T., Langenberg, C., Forouhi, N. G., Sharp, S., et al. (2011). Mediterranean diet and type 2 diabetes risk in the European Prospective Investigation into Cancer and Nutrition (EPIC) study. The InterAct project. Diabetes Care, 34, 1913-1918. doi:10.2337/dc11-0891.

Rosenthal, R., Rosnow, R. L. \& Rubin, D. B. (2000). Contrasts and effect sizes in behavioral research. A correlational approach. New York: Cambridge University Press.

Roskam, A.-J. R., \& Kunst, A. E. (2008). The predictive value of different socio-economic indicators for overweight in nine European countries. Public Health Nutrition, 11, 1256-1266. doi:10.1017/s1368980008002747.

Shepherd, R. (2002). Resistance to changes in diet. Proceedings of the Nutrition Society, 61, 267-272. doi:10.1079/pns2002147.

Shepperd, J. A., Klein, W. M. P., Waters, E. A., \& Weinstein, N. D. (2013). Taking stock of unrealistic optimism. Perspectives on Psychological Science, 8, 395-411. doi:10.1177/1745691613485247.

Sparks, P., Shepherd, R., Wieringa, N., \& Zimmermanns, N. (1995). Perceived behavioral control, unrealistic optimism and dietary change. An exploratory study. Appetite, 24, 243-255.

Sproesser, G., Strohbach, S., Schupp, H., \& Renner, B. (2011). Candy or apple? How self-control resources and motives impact dietary healthiness in women. Appetite, 56, 784-787. doi:10.1016/j.appet.2011.01.028.

Statistisches Bundesamt (2014a). Zahlen und Fakten. Available from <https:// www.destatis.de/DE/ZahlenFakten/ZahlenFakten.html> Last accessed 21.02.14.

Statistisches Bundesamt (2014b). Informationssystem der Gesundheitsberichterstattung des Bundes. Available from <https://www .gbe-bund.de/> Last accessed 21.02.14.

Taylor, S. E., \& Brown, J. D. (1988). Illusion and well-being. A social psychological perspective on mental health. Psychological Bulletin, 103, 193-210. doi:10.1037/ 0033-2909.103.2.193.

Van Boven, L., \& Loewenstein, G. (2003). Social projection of transient drive states. Personality and Social Psychology Bulletin, 29, 1159-1168. doi:10.1177/ 0146167203254597.

Weinstein, N. D. (1980). Unrealistic optimism about future life events. Journal of Personality and Social Psychology, 39, 806-820. doi:10.1037/0022-3514.39.5 806.

Weinstein, N. D. (1982). Unrealistic optimism about susceptibility to health problems. Journal of Behavioral Medicine, 5, 441-460. doi:10.1007/BF00845372.

WHO (2008). World health statistics 2008. Geneva, Switzerland: World Health Organization.

Wilcox, S., \& King, A. C. (2000). Self-favoring bias for physical activity in middle-aged and older adults. Journal of Applied Social Psychology, 30, 1773-1789. doi:10.1111/ j.1559-1816.2000.tb02467.x.

Winkler, G., Döring, A., \& Keil, U. (1991). Differences in dietary intake between weekend and weekdays. Results from the MONICA-project Augsburg dietary survey 1984/85. Zeitschrift für Ernährungswissenschaft, 30, 313-317.

Winkler, G., \& Döring, A. (1995). Kurzmethoden zur Charakterisierung des Ernährungsmusters. Einsatz und Auswertung eines Food-Frequency-Fragebogens [Brief methods for descr bing eating patterns. Application and analysis of a food frequency questionnaire]. Ernährungsumschau, 42, 289-291.

Winkler, G., \& Döring, A. (1998). Validation of a short qualitative food frequency list used in several German large scale surveys. Zeitschrift für Ernährungswissenschaft, $37,234-241$. 